\title{
PRELIMINARY RESULTS FROM THE PORTABLE IMAGERY QUALITY ASSESSMENT TEST FIELD (PIQUAT) OF UAV IMAGERY FOR IMAGERY RECONNAISSANCE PURPOSES
}

\author{
R. Dabrowski a ${ }^{\text {, A. Orych }}{ }^{\text {a }}$, A. Jenerowicz ${ }^{\text {a }}$, P. Walczykowski ${ }^{\text {a }}$, \\ a MUT Military University of Technology, Faculty of Civil Engineering and Geodesy, Geodesy Institute, Department of Remote \\ Sensing and Photogrammetry, - (rafal.dabrowski, agata.orych, agnieszka.jenerowicz, piotr.walczykowski)@wat.edu.pl
}

KEY WORDS: UAV, IMINT, Quality assessment Test Field, Remote sensing, miniMCA

\begin{abstract}
:
The article presents a set of initial results of a quality assessment study of 2 different types of sensors mounted on an unmanned aerial vehicle, carried out over an especially designed and constructed test field. The PIQuAT (Portable Imagery Quality Assessment Test Field) field had been designed especially for the purposes of determining the quality parameters of UAV sensors, especially in terms of the spatial, spectral and radiometric resolutions and chosen geometric aspects. The sensor used include a multispectral framing camera and a high-resolution RGB sensor. The flights were conducted from a number of altitudes ranging from $10 \mathrm{~m}$ to 200 $\mathrm{m}$ above the test field. Acquiring data at a number of different altitudes allowed the authors to evaluate the obtained results and check for possible linearity of the calculated quality assessment parameters. The radiometric properties of the sensors were evaluated from images of the grayscale target section of the PIQuAT field. The spectral resolution of the imagery was determined based on a number of test samples with known spectral reflectance curves. These reference spectral reflectance curves were then compared with spectral reflectance coefficients at the wavelengths registered by the miniMCA camera. Before conducting all of these experiments in field conditions, the interior orientation parameters were calculated for the MiniMCA and RGB sensor in laboratory conditions. These parameters include: the actual pixel size on the detector, distortion parameters, calibrated focal length (CFL) and the coordinates of the principal point of autocollimation (miniMCA - for each of the six channels separately.
\end{abstract}

\section{INTRODUCTION}

In the past few years there had been a significant increase in the availability of imagery data, especially that obtained from sensors placed on unmanned aerial vehicles (Bendig et al., 2012) for remote sensing purposes, also for imagery reconnaissance. One of the most important aspects of acquiring imagery data for IMINT purposes is knowing what quality of imagery the utilized UAV system configuration is capable of acquiring. Many image artefacts and errors are not only dependant on the sensor itself, but also can vary depending on the type of the utilized UAV system. The quality of received data (which is directly linked to the accuracy of the final product upon which they had been created) is determined by many factors. The most important of them include: the stability of the flight path of the platform, the flight altitude, and the quality of the sensor itself. The quality of the system is determined by the spatial and radiometric resolution of the image and aberrations with which they are burdened (Kelcey \& Lucieer, 2012) This is why such research should be carried out not only on the sensors in laboratory conditions, but on the complete UAV system configuration, incorporating the aerial vehicle itself, the sensors and any additional components in real life field conditions. The most common solution in the field conditions are calibration tests. The term calibration test is an especially designed structure, which when laid out flat on the ground and photographed, can be used to evaluate the sensor's imaging capabilities. Each target has very specific geometrical and spectral characteristics. Such structures usually have a flat modular design with the geometrical calibration elements applied with paints. These elements have very carefully designed spatio-frequency characteristics. Determining the ground resolved distance is based on analyses of images of these elements in terms of the amount of information (details) which had been transferred from the actual target, via the atmosphere, the optical system and imaging system, to the final image. There is a great number of calibration targets used, their size and shape depending on their expected use ( Honkavaara et al., 2008; Honkavaara et al., 2010).

The article presents a set of initial results of a quality assessment study of two different types of sensors mounted on an unmanned aerial vehicle, carried out over an especially designed and constructed test field

\section{UAV SYTEM}

The UAV system used in the experiment consist of two components: UAV platform and imagery sensors.

\subsection{Sensors}

Two different sensor were used in the experimentt: miniMCA multispectral framing camera and Sony Nex-5 high-resolution RGB camera.

\subsection{1 miniMCA}

The compact multispectral miniMCA camera has been specially designed to be mounted on board an unmanned aerial vehicle. The camera weighs 700 grams and its dimensions are $114 \times 78$ $\mathrm{x} 80 \mathrm{~mm}$. The tested camera was equipped with six separate CMOS sensors effective spectral range of data acquisition of 400 - $950 \mathrm{~nm}$. The part of the EM spectrum which will be imaged is determined by the applied interference filters . The camera uses standard interference filters with a diameter of 25 $\mathrm{mm}$. It is possible to replace the cameras filters. The tested camera uses filters with values $\lambda \max =550,490,610,660,720$, $850 \mathrm{~nm}$. The matrix resolution of each sensor is $1280 \times 1024$ 
with a pixel size of 5.2 microns. The radiometric resolution is 10-bit.

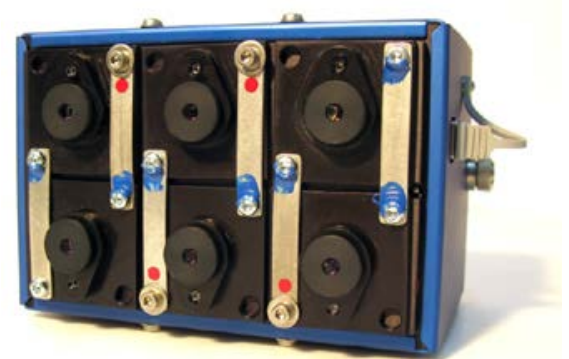

Figure 1. miniMCA multispectral camera

The camera uses a standard USB interface for communication and control of operations. An additional and very important advantage of the camera MCA 6 is its ability to collect data directly to a memory card. For registering data from each sensor a data card with a capacity of 2 GB can be used, which gives an effective memory of up to $12 \mathrm{~GB}$. The camera also allows you to connect an external GPS receiver[4]. Before executing experiment the interior orientation parameters were calculated for the MiniMCA sensor in laboratory conditions based on the 3D and 2D test fields. The interior orientations parameters are shown in (Dabrowski et al., 2013)

\subsubsection{Sony Nex-5}

The Sony NEX-5 is a digital camera launched on 11 May 2010. It is a mirrorless interchangeable lens camera with the body of a common point-and-shoot camera but with a sensor size (APSC) comparable to that of some digital single-lens reflex cameras. The NEX-5 shoots 14.2 megapixel stills and has a 7 frame/s continuous shot mode. It has the capability to shoot $1920 \times 1080 \mathrm{i}$ at $60 \mathrm{frame} / \mathrm{s}$ in AVCHD or $1440 \times 1080 \mathrm{p}$ at 30 frame/s in MPEG4. Due to its low tare weight (502 grams) and high image quality parameters, the camera excels as a UAV sensor.

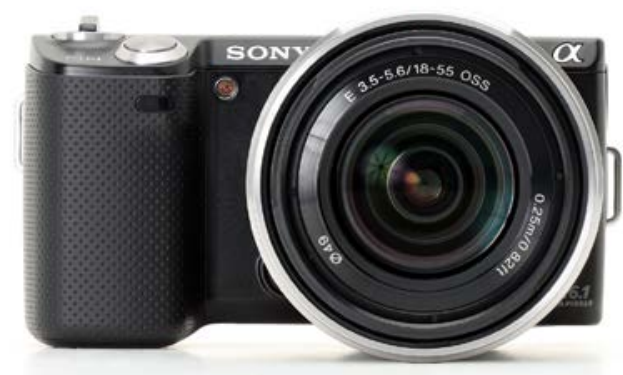

Figure 2. Sony Nex-5 RGB camera [sony.pl]

\subsection{UAV platform}

Unmanned aircraft used as a carrier for listed up sensors was German Rotorcraft MicroCopter. This UAV has the ability to conduct automated flight after previously preset points along the route. UAV maximum operating radius is approximately $700 \mathrm{~m}$, height approximately $500 \mathrm{~m}$ and operational capacity is $2.5 \mathrm{~kg}$.

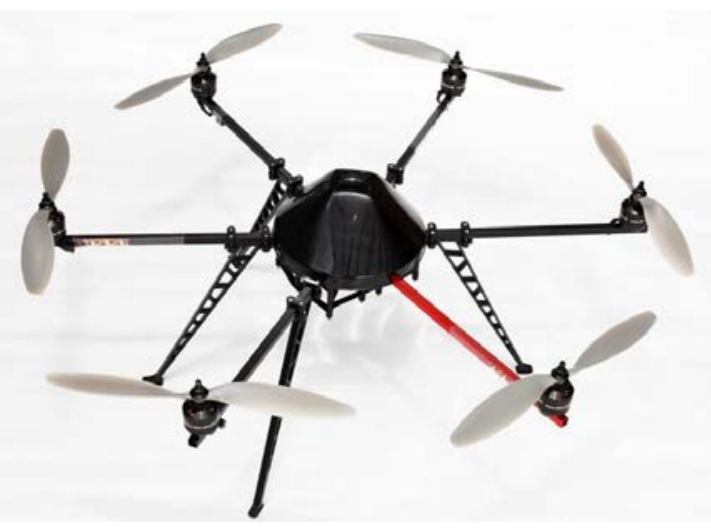

Figure 3. Octocopter [mikrokopter.de]

\section{PORTABLE IMAGERY QUALITY ASSESSMENT TEST FIELD}

The PIQuAT (The Portable Imagery Quality Assessment Test Field) is the portable test field, dedicated for different sensors mounted on UAVs that allows for evaluation of the quality of images. The PIQuAT, designed and build by research team from Military University of Technology, Faculty of Civil Engineering and Geodesy, Geodesy Institute, Department of Remote Sensing and Photogrammetry, consists of 6 individual segments, when combined allow for determine radiometric, spectral and spatial resolution of images acquired from UAVs. All segments of the PIQuAT can be used together in various configuration or independently. First segment is used to determine the spatial resolution of UAVs' sensors and consists of a quarter of the 32 sectorial Siemens Star. The next three segments of the test together form a 12- level grayscale board (from black- 3\% reflectance to white- 85\% reflectance) for evaluation of radiometric resolution. The last two parts are used for assessment of sensor's spectral resolution- first segment is covered with four different types of materials- wood, red PVC, aluminium plate and green textile. The second segment used for assessment of spectral resolution is divided into four parts- two are painted with two different kinds of green paint and the next two with two kinds of red paint. Both red and green colours are almost indistinguishable with the naked eye or RGB sensor, but additional spectral channel, e.g. IR would allow to distinguish them. More information about the PIQuAT can be found in the article "Portable Imagery Quality Assessment Test Field for UAV sensors” (R. Dabrowski, A. Jenerowicz, 2015).

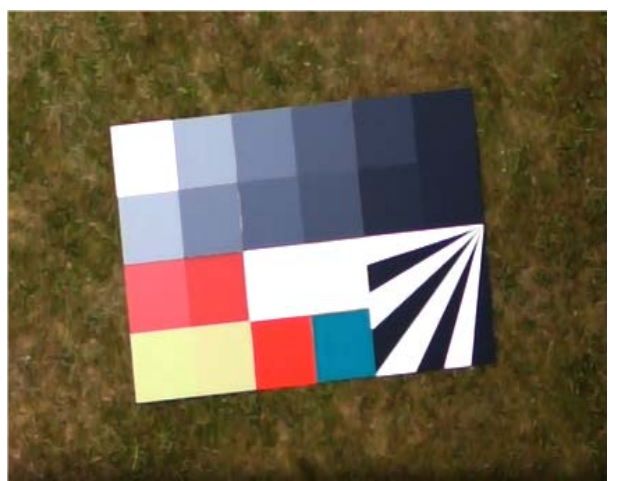

Figure 4a. Part of the RGB PIQuAT image aquired from RGB camera. 


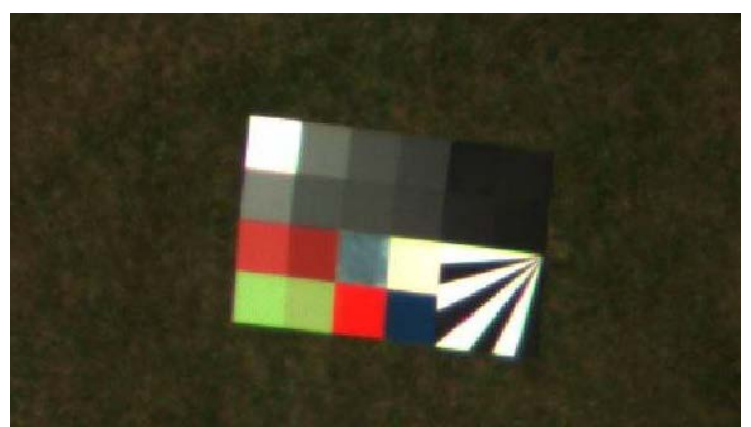

Figure 4b. Part of the RGB PIQuAT image aquired from miniMCA camera.

\section{EXPERIMENT}

The research experiment consisted of series of UAV flights at different altitudes- every 10 meters, from 10 to 200 meters over the test field and acquisition of images with different sensors. Before performing test flights, there were determined elemet of interior orientations of the cameras in the laboratory conditions. In addition to that for the camera miniMCA there were determined optimal exposure conditions, that the values DN of white reflectance panel (98\%) stood at $75 \% \mathrm{DN}_{\max }$. (Dabrowski \& Orych, 2014).

These data were used to plan the UAV flight parameters in terms of determining GSD parameter and spatial coverage of cameras' frames. After acquisition of image data they were post processed in PW2 and Erdas Imagine software, and then analyzed. First, the GRD parameter was analyzed. On the basis of the part of the Siemens star, the group of 10 analysts measured the radius of blurred spot, what allowed for determination of GRD. This parameter is strongly correlated with the potential of photo interpretation of imagery data and their application in imagery intelligence.

The next phase of the experiment involved the extraction of spectral information from images acquired with the camera miniMCA. During the analysis process, it was assumed that the DN value of pixels directly corresponds to the spectral reflectance. Prior to the extraction of spectral information of individual pieces of the PIQuAT, all data were processed with the assumption that used reflectance pattern (located next to the test) is characterized by a reflectance of $98 \%$. The obtained information was compared to the spectral reflectance characteristics of each field of PIQuAT obtained in laboratory conditions using the spectroradiometer.

\section{RESULTS}

\subsection{The geometric aspect}

In the case of the camera Sony Nex- 5 the parameter GRD is changing linearly with height and varies between $0.01 \mathrm{~m}$ to 0.07 $\mathrm{m}$ for the flight altitude of $170 \mathrm{~m}$. In the Figure 5 it is presented the averaged GRD parameter as a function of flight altitude, along with the theoretical GSD values.

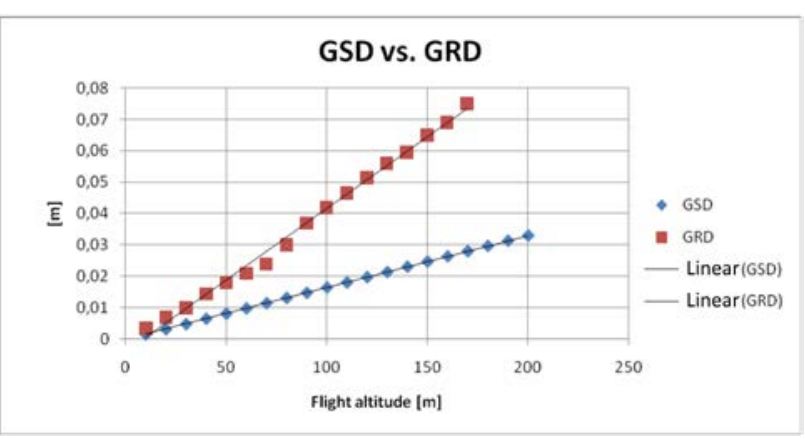

Figure 5. GSD vs GRD - for the camera Sony Nex-5

Determination of GRD parameter on the basis of images obtained from higher altitudes was impossible due to parameters of Siemens star used in the PIQuAT. When analyzing the imagery data from successive UAV flights it can be seen that the greatest errors occurred in images acquired from 70-90m. This errors occurred due to dynamic changes of light conditions (cloud cover), which resulted in difficulties with the exact length measurement on Siemens star. For camera miniMCA each channel was analyzed separately. As in the case of the camera Sony Nex 5, obtained GRD parameter changes for each channel in a linear way with changes of altitude and varies in the range from 0.01 to $0.075 \mathrm{~m}$ for an altitude of $70 \mathrm{~m}$. The biggest difference in the GRD parameter (between channels Master and Slave 1) occurred in the pictures obtained from a height of $30 \mathrm{~m}$. However, the value of this difference was equal to $0.002 \mathrm{~m}$, which demonstrate that camera should be considered as homogenous in this aspect. Figure No. 6 presents the averaged GRD parameter as a function of altitude, along with the theoretical GSD values.

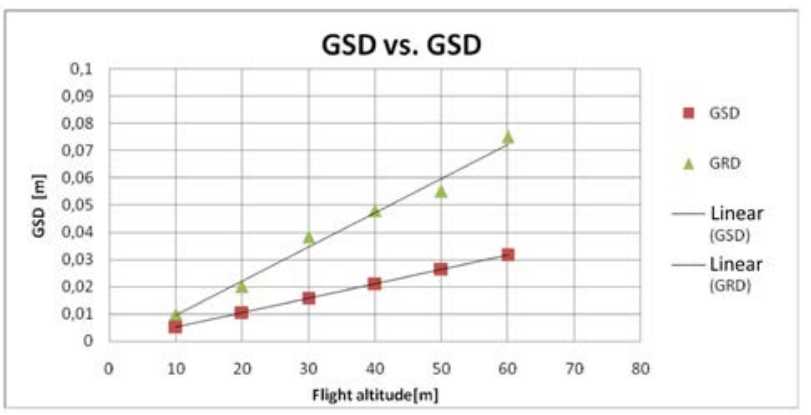

Figure 6.GSD vs GRD - for the camera miniMCA

All differences between analysed cameras in the GRD parameter are caused by different array size and different focal length of investigated cameras.

\subsection{The radiometric aspekct}

When analysing imagery data obtained with the camera Sony Nex- 5, it can be seen, that camera correctly presented the second segment of the PIQuAT- which is used for evaluation of radiometric resolution, and presents 12- level greyscale. However, with the different spectral resolution and camera sensitivity than miniMCA, it is impossible to distinguish elements covered with paint green 1 and green 2, and elements covered with aluminium plate and wood. In the different colour compositions from miniMCA it is possible to distinguish all mentioned elements. 


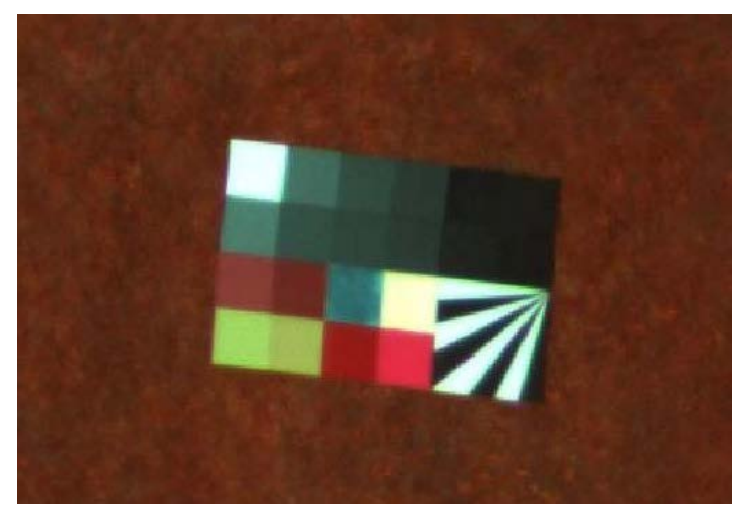

Figure 7. Part of the NIR, G, B band composition of PIQuAT image

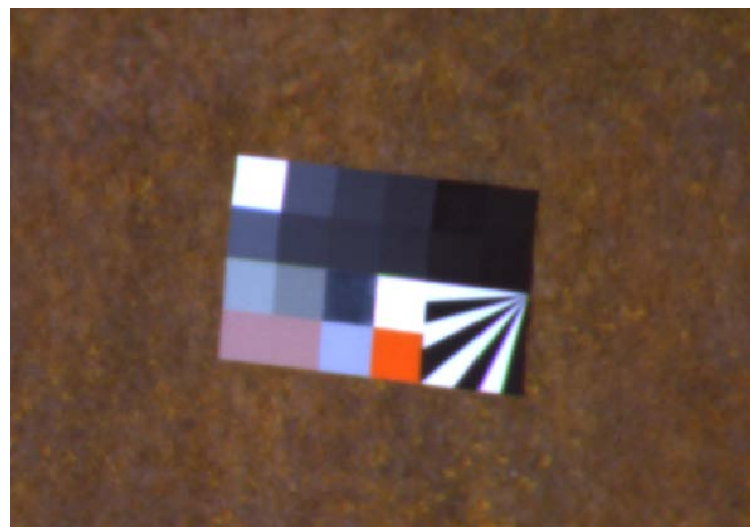

Figure 8. Part of the NIR, NIR, R band composition of PIQuAT image

On the basis of imagery data the spectral characteristics of chosen elements of the PIQuAT were obtained. In the Figure 9, it are shown spectral characteristics of grey panels, where the shade of gray are changing with $10 \%$ step. In the Figure 10 is shown the spectral reflectance characteristics of the same fields, but obtained with spectroradiometer under the laboratory conditions. When analyzing the above graphs, it can be seen that both curves have similar shape but different reflectance values. These differences sniffing is in the range of $6 \%$ for darkest fields and grows up to $16 \%$ for lightest fields.

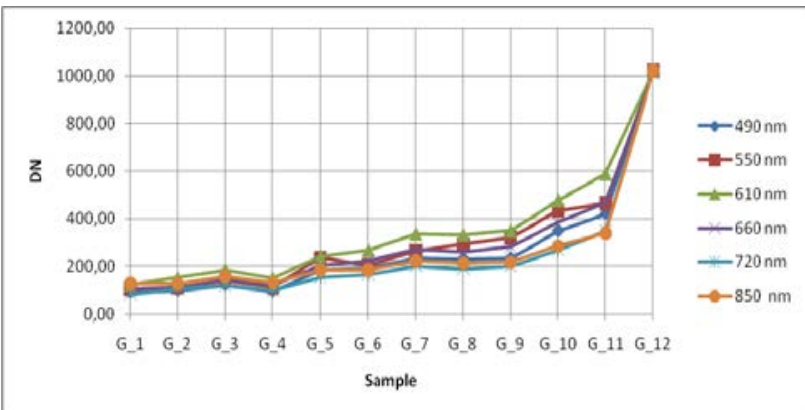

Figure 9. Spectral characteristics acquired from minMCA images

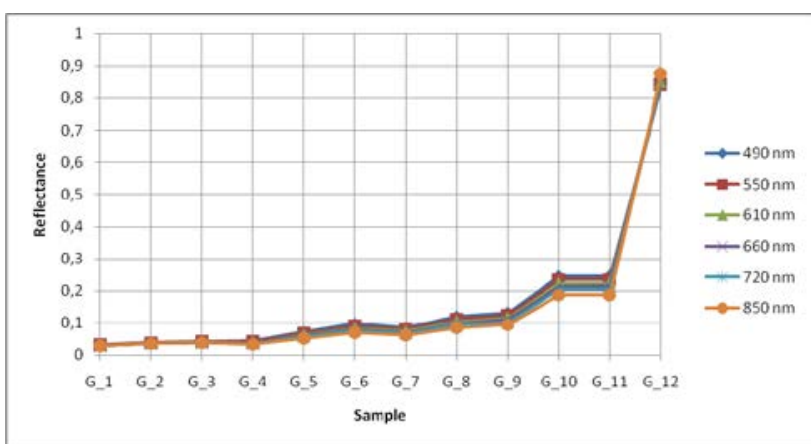

Figure 8. Spectral characteristics acquired from spectroradiometer

The obtained values are very promising and are the basis for further research especially in the area of the accuracy improvement of the determination of the spectral reflectance coefficients on a basis of imagery data from multispectral camera miniMCA without the usage of reflectance panel.

\section{CONCLUSIONS}

All results included in the article are only preliminary results of the initial stage of conducted analysis of imagery data obtained with sensors mounted on UAVs. However, it is possible to say that the image data obtained with the camera Sony Nex 5 and the minMCA have a high potential of photo interpretation and are complementary in the field of image intelligence. With high spatial resolution data acquired from NEX 5 and a relatively high spectral resolution of data from the camera miniMCA, the integration of imagery data allows not only for imagery intelligence but also for analysis in precision agriculture and in crisis management systems.

\section{ACKNOWLEDGEMENTS}

The presented article is part of research work carried out in the "Innovative remote sensing system for the monitoring of pollutants in rivers, offshore waters and flooded areas“ project PBS1/B9/8/2012.

\section{REFERENCES}

J. Bendig , A. Bolten, G. Bareth, „Introducing a low-cost miniUAV For thermal- and multispectral-imaging”, International Archives of the Photogrammetry, Remote Sensing and Spatial Information Sciences, Volume XXXIX-B1, 2012 XXII ISPRS Congress, 25 August - 01 September 2012, Melbourne, Australia.

J. Kelcey, A. Lucieer, „Sensor correction and radiometric calibration of a 6-band multispectral imaging sensor for uav remote sensing”, International Archives of the Photogrammetry, Remote Sensing and Spatial Information Sciences, Volume XXXIX-B1, 2012 XXII ISPRS Congress, 25 August - 01 September 2012, Melbourne, Australia.

Honkavaara, E., Peltoniemi, J., Ahokas, E., Kuittinen, R., Hyyppä, R., Jaakkola, J., Kaartinen, H., Markelin, L., Nurminen, K., Suomalainen J., 2008. A permanent test field for 
The International Archives of the Photogrammetry, Remote Sensing and Spatial Information Sciences, Volume XL-1/W4, 2015 International Conference on Unmanned Aerial Vehicles in Geomatics, 30 Aug-02 Sep 2015, Toronto, Canada

digital photogrammetric systems. Photogrammetric Engineering \& Remote Sensing, volume 74, number 1, pp. 95-106

Honkavaara, E., Hakala, T., Peltoniemi, J., Suomalainen, J., Ahokas, E., Markelin, L., 2010. Analysis of Properties of Reflectance Reference Targets for Permanent Radiometric Test Sites of High Resolution Airborne Imaging Systems. Remote Sens. 2010, 2, pp. 1892-1917

R. Dąbrowski, A. Orych, P. Walczykowski, "Evaluation of the possibility of using the miniMCA multispectral camera in Imagery IntelligenceResearch" Conference In Technical Disciplines, November, 18. - 22. 2013 